\title{
Posterior fixation without debridement for vertebral body osteomyelitis and discitis
}

\author{
Ahmed S. Mohamed, M.D., ${ }^{1}$ Jung Yoo, M.D., ${ }^{1}$ Robert Hart, M.D.,${ }^{1}$ Brian T. Ragel, M.D.,${ }^{2}$ \\ Jayme Hiratzka, M.D., ${ }^{1}$ D. Kojo Hamilton, M.D., ${ }^{2}$ Penelope D. Barnes, Ph.D., M.B.B.S., ${ }^{2}$ \\ and Alexander C. Ching, M.D. ${ }^{1}$ \\ Departments of ${ }^{1}$ Orthopedics and Rehabilitation and ${ }^{2}$ Neurological Surgery, Oregon Health \& Science University, \\ Portland, Oregon
}

Object. The authors evaluated the efficacy of posterior instrumentation for the management of spontaneous spinal infections. Standard surgical management of spontaneous spinal infection is based on debridement of the infected tissue. However, this can be very challenging as most of these patients are medically debilitated and the surgical debridement requires a more aggressive approach to the spine either anteriorly or via an expanded posterior approach. The authors present their results using an alternative treatment method of posterior-only neuro-decompression and stabilization without formal debridement of anterior tissue for treating spontaneous spinal infection.

Methods. Fifteen consecutive patients were treated surgically by 2 of the authors. All patients had osteomyelitis and discitis and were treated postoperatively with intravenous antibiotics for at least 6 weeks. The indications for surgery were failed medical management, progressive deformity with ongoing persistent spinal infection, or neurological deficit. Patients with simple epidural abscess without bony instability were treated with laminectomy and were not included in this series. Fourteen patients were treated with posterior-only decompression and long-segment rigid fixation, without formal debridement of the infected area. One patient was treated with staged anterior and posterior surgery due to delay in treatment related to medical comorbidities. The authors examined as their outcome the ambulatory status and recurrence of deep infection requiring additional surgery or medical treatment.

Results. Of the initial 15 patients, $10(66 \%)$ had a minimum 2-year follow-up and 14 patients had at least 1 year of followup. There were no recurrent spinal infections. There were 3 unplanned reoperations ( 1 for loss of fixation, 1 for early superficial wound infection, and 1 for epidural hematoma). Nine (60\%) of 15 patients were nonambulatory at presentation. At final followup, 8 of 15 patients were independently ambulatory, 6 required an assistive device, and 1 remained nonambulatory.

Conclusions. Long-segment fixation, without formal debridement, resulted in resolution of spinal infection in all cases and in significant neurological recovery in almost all cases. This surgical technique, when combined with aggressive antibiotic therapy and a multidisciplinary team approach, is an effective way of managing serious spinal infections in a challenging patient population.

(http://thejns.org/doi/abs/10.3171/2014.6.FOCUS14142)

\section{KEY WoRDS $\bullet \quad$ de novo spinal infection $\bullet \quad$ single stage procedure $\bullet \quad$ spondylodiscitis $\bullet$
posterior spinal fixation $\bullet \quad$ osteomyelitis}

I $\mathrm{N}$ the adult population, vertebral discitis and osteomyelitis can be challenging problems to treat. Many patients are immunocompromised and often nutritionally deficient. ${ }^{11}$ The majority of these patients can be treated with a 6- to 8-week course of intravenous antibiotics. ${ }^{12}$ In select cases, however, epidural abscess results in either impending or actual neurological deficit. In other cases, progressive bony destruction leads to structural instability that requires surgical treatment. The traditional gold standard of surgical management for these infections has involved aggressive debridement of the infected area with structural reconstruction. ${ }^{1}$ This technique is effective but can result in increased morbidity in this patient population. The reported mortality after surgical treatment is between $8 \%$ and $14 \% .^{1,11}$ We present a case series of patients managed with posterior fixation and decompression, without formal debridement of the

Abbreviation used in this paper: $\mathrm{BMP}=$ bone morphogenetic protein. infected area. The rationale for this approach is that rigid stabilization across the infected involved vertebral body and disc space will allow better antibiotic penetration and tissue healing, similar to what has been postulated in the literature on open tibia fractures regarding the use of bone morphogenetic proteins (BMPs).

\section{Methods}

After receiving institutional review board approval, we reviewed our surgical case database for adult patients treated surgically for active de novo spinal infection by 2 authors (A.C.C. and J.Y.) between 2007 and 2011. Patients with epidural abscess without spinal instability were treated with laminectomy only and were not included in the current case series. All patients included in this series were being treated for active infection; patients being treated for the late sequelae of osteomyelitis, such as residual stenosis or late deformity but without ongoing infection, were not included. Patients with postoperative infection were 
excluded. We performed a retrospective chart review to identify treatment failures and complications.

\section{Patient Outcomes}

Treatment failure was defined as a recurrent need for medical care for an infectious condition in the spine. We also tracked any recurrent positive blood cultures, repeat hospitalizations for any infectious diagnoses, and any further spine surgery, either at the site of the index infection or a remote site. Patients returned for close clinical follow-up, in most cases in a multidisciplinary spine surgery and infectious disease clinic, for at least the first 6 months after surgery. Due to the poor specificity of inflammatory markers (erythrocyte sedimentation rate and C-reactive protein) and the common comorbidities in this population, we did not routinely follow these markers after stopping intravenous antibiotics. Instead, our policy was to monitor these patients clinically. If new symptoms appeared (pain, new neurological deficit, fever or other flu-like symptoms, or wound drainage) we obtained repeat advanced imaging and a full set of laboratory tests.

\section{Results}

We identified 15 patients who met the inclusion criteria. Diagnoses included discitis and osteomyelitis with and without epidural abscess. Indications for initial surgery included failure of prolonged medical management, progressive bony collapse or instability, and/or presence of epidural abscess causing neurological compression (Table 1). In most cases, patients had more than 1 of these indications for surgery; neurological deficit was the most common indication (12 patients [80\%]). Seven patients $(47 \%)$ presented with acute infection and progressive neurological deficit; these patients underwent decompression and stabilization as the initial management. Treatment was attempted in 8 patients (53\%) prior to stabilization. Five of these patients initially had a laminectomy and antibiotic therapy before developing documented instability and recurrence of infection, resulting in a repeat operation that included a stabilization procedure (Table 2). Five patients underwent initial nonoperative treatment for known spinal infection that went on to failure. Two of these patients presented with acute neurological deterioration during antibiotic therapy and underwent emergency decompression and stabilization. Three patients were treated with prolonged antibiotic therapy but had progressive bony destruction, persistent fevers, and elevated inflammatory markers and were then indicated for definitive fixation. All but 2 of our patients had spinal instability defined either by progressive collapse/pathological fracture of at least 1 vertebral body, gross motion on dynamic lateral radiographs, or gross instability during surgical exploration for laminectomy. Two patients did not have defined instability at presentation, but after aggressive decompression the patients were felt to be at risk for sagittal deformity.

All patients were treated with intravenous antibiotics under the management of an infectious disease specialist (see Table 3 for duration and type of antibiotic therapy). Eleven patients had their antibiotic therapy managed as part of our multidisciplinary clinic and had complete antibiotic
TABLE 1: Characteristics in 15 patients who underwent posterior fixation for vertebral body osteomyelitis and discitis*

\begin{tabular}{lc}
\hline \multicolumn{1}{c}{ Parameter } & Value† \\
\hline mean age in yrs & $59 \pm 10.4$ \\
sex distribution & $80 \% \mathrm{M}, 20 \% \mathrm{~F}$ \\
mean Charlson Comorbidity Index & 2 \\
mean ASA score & 2.8 \\
prior spine surgery & $60 \%$ \\
mean BMI & $29.5 \pm 8.1$ \\
average preop albumin in g/dl & 2.1 \\
preop neurological deficit & $12(80)$ \\
bony instability & $13(86)$ \\
failure of prior treatment & $8(53)$ \\
\hline
\end{tabular}

* $\mathrm{ASA}=$ American Society of Anesthesiologists; $\mathrm{BMI}=$ body mass index.

$\dagger$ Values are presented as the number of patients (\%) unless stated otherwise. Mean values are presented as means \pm SD.

data. Three patients had infectious disease management in an outside medical system, and their antibiotic therapy data were incomplete, and 1 patient was lost to follow-up before a definitive antibiotic plan was established.

Fourteen patients were treated with posterior-only, long-segment rigid fixation, without formal debridement of the infected material. One patient was treated with staged posterior and then anterior surgery. All patients had at least 2 levels of fixation above the involved disc space (average number of levels 2.7), including both hooks and screw fixation. Below the affected disc space, all patients had at least 2 levels of pedicle fixation (average 2.7), with the exception of the 4 patients with iliac fixation (which was regarded as fixation equivalent to 2 levels of pedicle screws). Neurological decompression was performed in all cases, regardless of presence of a neurological deficit. In 3 cases (20\%), the disc space was at least minimally debrided or cultured through a posterior approach. The vast majority of patients (12 of 15) had formal posterolateral fusion, usually with allograft and recombinant BMP (Table 2). Three patients had percutaneous fixation without formal attempt at fusion; all of these patients underwent subsequent hardware removal between 10 and 14 months after fixation. One patient underwent open fusion at L1-5, with open fixation at L1-S1, and had selective removal of S-1 hardware at 1 year after surgery for the resulting (expected) L5-S1 nonunion. Figures 1-3 show the initial images, postoperative radiographs, and a late follow-up CT scan for this patient.

The median follow-up was 35 months (range 0.2-66 months). Fourteen patients underwent follow-up longer than 12 months. One patient was lost to follow-up early after surgery. There were no recurrent deep spinal infections in any of the 14 patients. No patient underwent a reoperation or new antibiotic therapy for spinal infection after the initial treatment course, with the exception of 1 superficial wound irrigation and debridement within 2 weeks of surgery.

There were 3 unplanned reoperations: 1 for loss of fixation of iliac hardware, 1 for new superficial wound 
Spinal infection

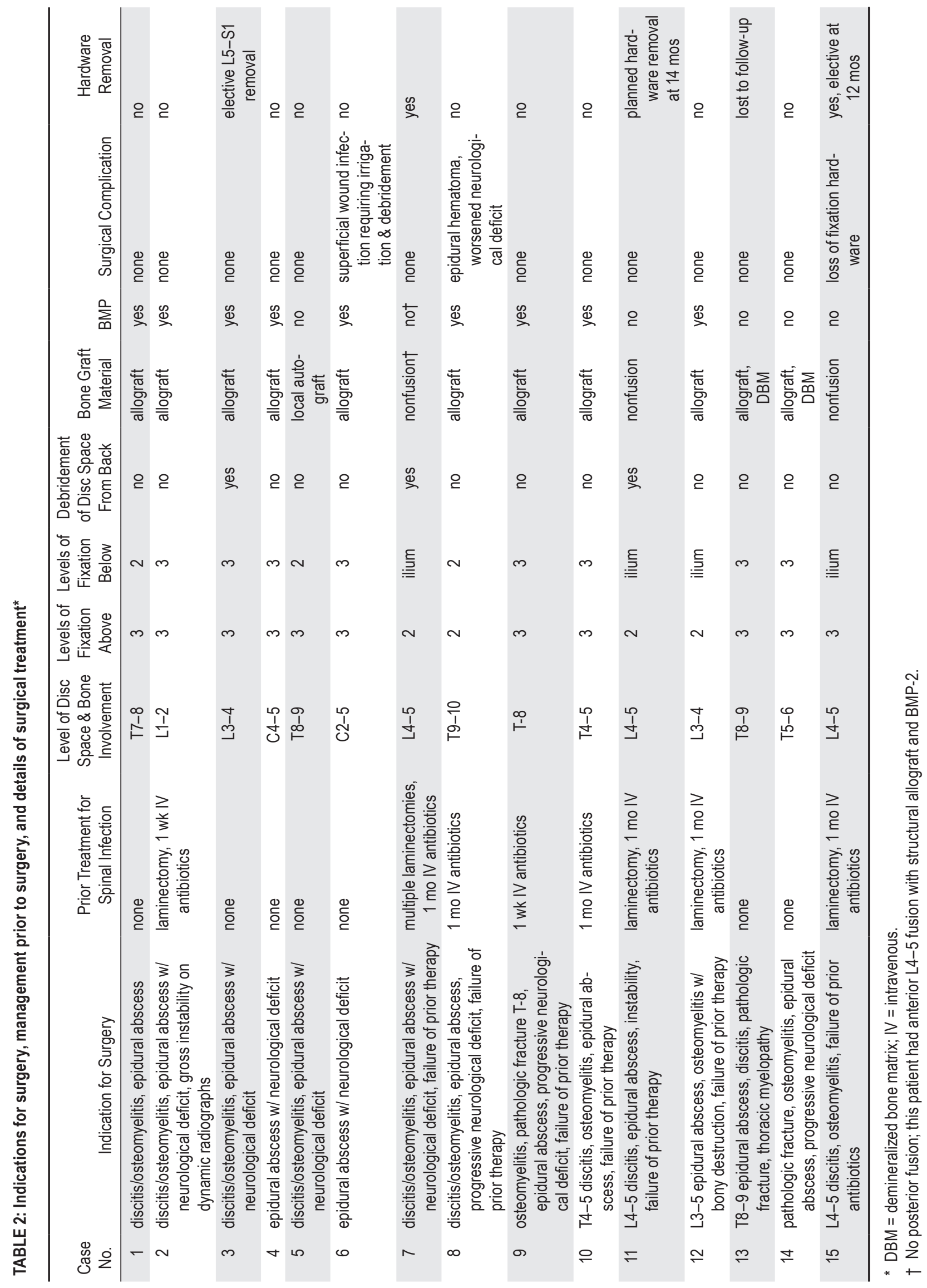


A. S. Mohamed et al.

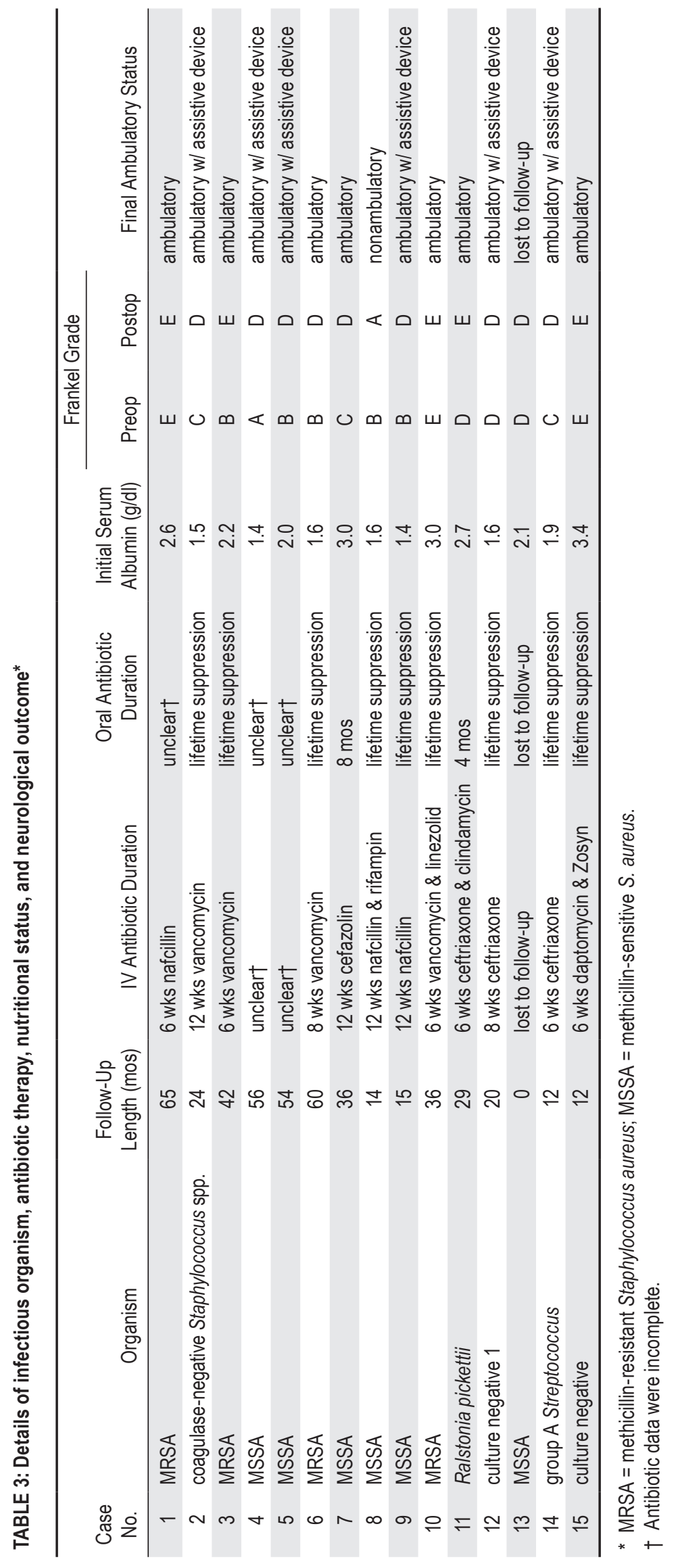



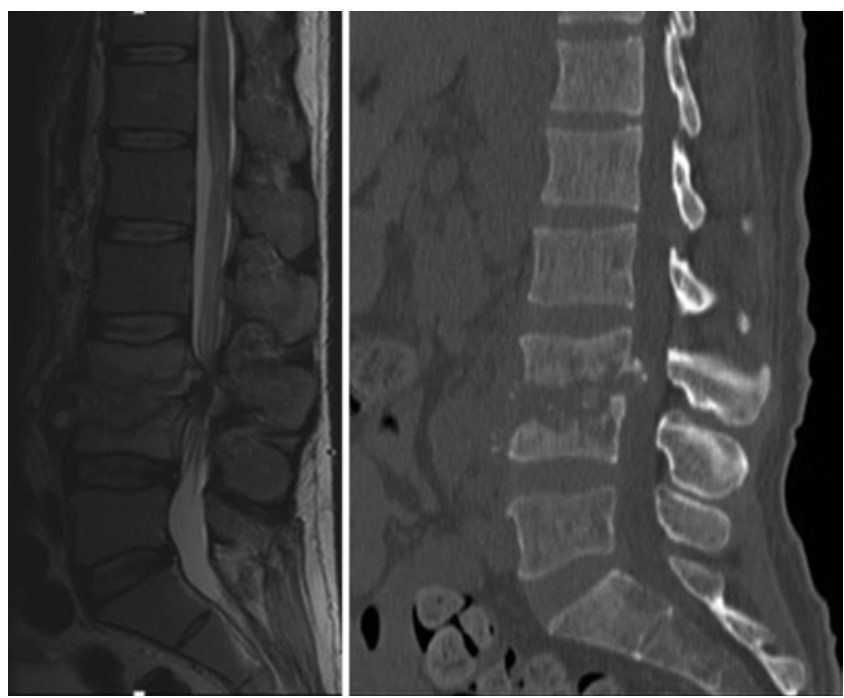

FIG. 1. Case 3. Preoperative MR image (left) and CT scan (right) showing discitis/osteomyelitis with bony destruction and instability, along with epidural abscess causing bilateral lower-extremity paraplegia.

infection, and 1 for epidural hematoma and neurological deterioration within 24 hours of index surgery. Ten (66\%) of 15 patients initially presented with a neurological deficit; the deficit in 9 patients $(60 \%)$ was such that they were nonambulatory at presentation. At final follow-up, 8 of 15 patients were independently ambulatory, 6 required an assistive device, and 1 remained nonambulatory. These ratios of ambulatory and nonambulatory patients compared between preoperative and postoperative were statistically significant $(\mathrm{p}=0.005)$.

Three patients died during follow-up of unrelated causes ( 2 of cancer and 1 of liver failure, all $>12$ months after surgery), and 1 patient died of unknown causes 58 months after surgery. Three patients had as their index procedure stabilization without fusion and therefore had planned repeat surgery for removal of the hardware. None of these patients had any evidence of infection at the time of hardware removal. We did not routinely assess fusion with advanced imaging, but no patient had symptomatic nonunion requiring intervention, aside from those who had planned hardware removal as described above.

\section{Discussion}

The important finding of this case series was that posterior long-segment fixation, without formal debridement, resulted in resolution of bacterial spinal infection in all cases.

Bacterial spinal infections include discitis, vertebral osteomyelitis, epidural abscess, and spondylitis and are most commonly caused by pyogenic bacteria such as Staphylococcus aureus. ${ }^{5}$ Classic teaching for the treatment of skeletal infections includes intravenous antibiotics, debridement of all pus, and avoiding hardware placement, if possible, for fear of bacterial colonization of the instrumentation. ${ }^{1}$ There are, however, multiple studies in the neurosurgical and orthopedic literature that have reported on patients with osteomyelitis, discitis, and ab-

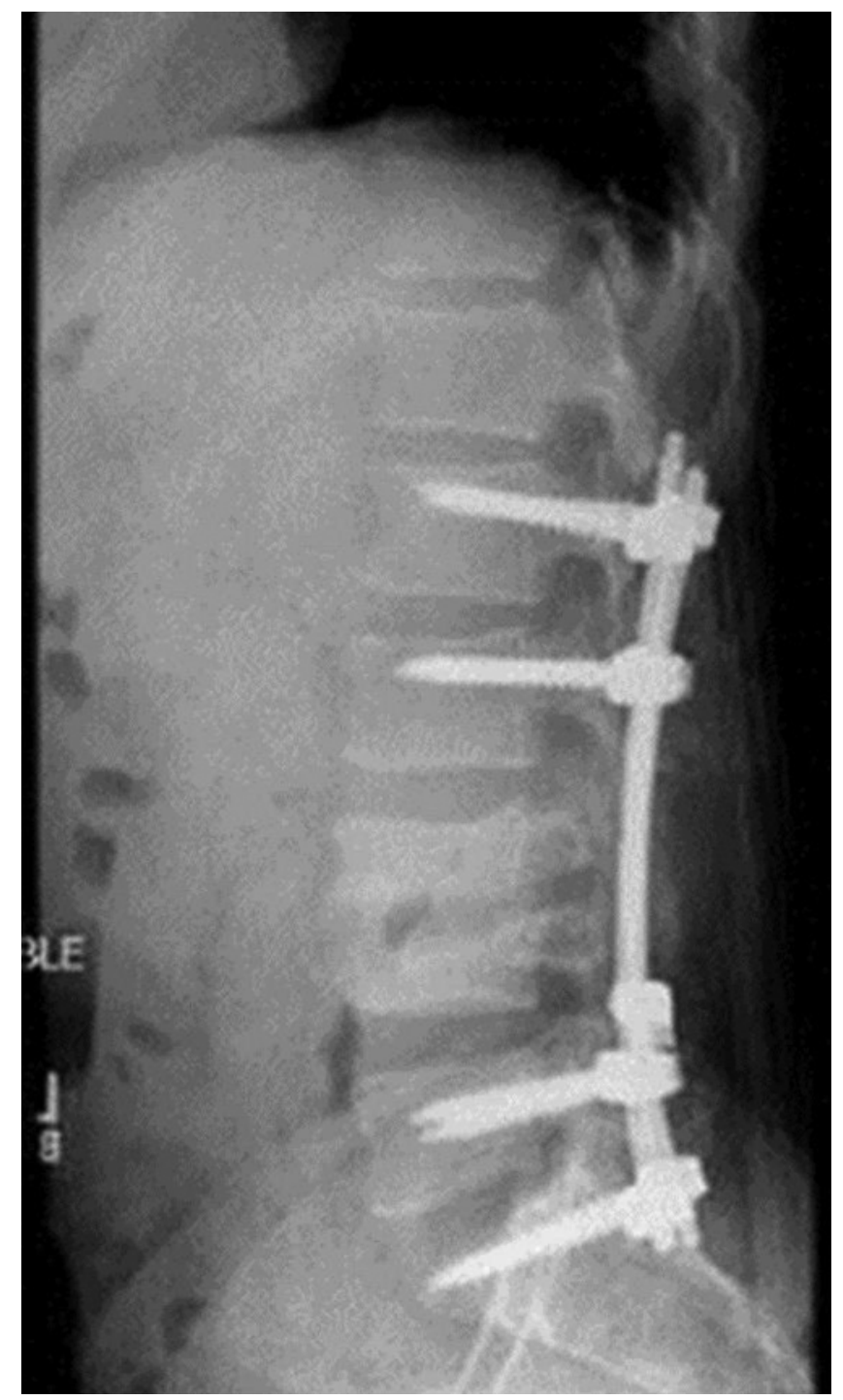

FIG. 2. Case 3. Initial postoperative radiograph obtained after posterior decompression of epidural abscess and stabilization of the infected segment.

scess who underwent spinal stabilization while acutely infected and had successful infection outcomes. In addition, the "acutely" instrumented patients are less likely to require additional surgery than patients who were not treated with spinal instrumentation. . $^{4,8}$

The best type and location of instrumentation for management of spine infections is unknown. Spine infections generally affect the anterior vertebral elements, thereby making the anterior surgical approach a more direct intervention to debride the infected material. However, the posterior approach is more attractive as it is associated with earlier mobilization, more rapid rehabilitation, and, in 1 study, higher fusion rates. ${ }^{3,4,6}$

Lee et al. found the posterior approach to be less advantageous in cases of severe bony destruction because posterior instrumentation may need to be extended over longer segments compared with the anterior approach.? Their study, however, involved tuberculous spondylodiscitis compared with more "typical" bacterial organisms 


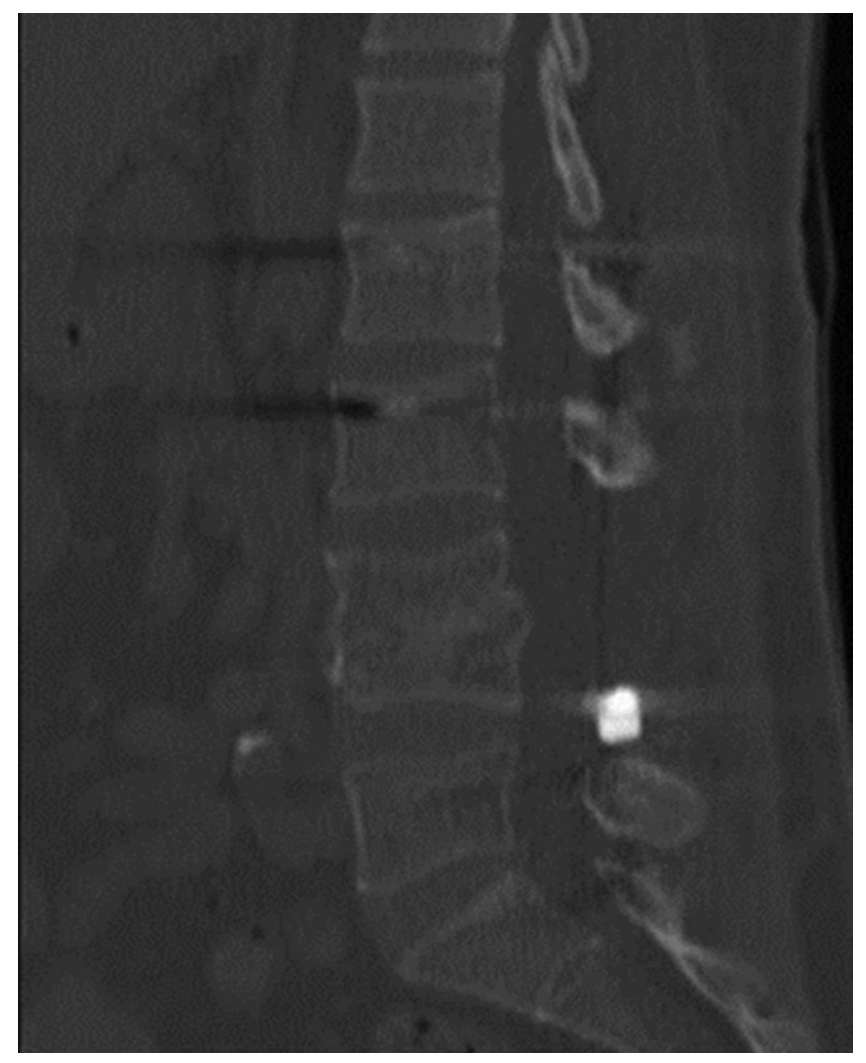

FIG. 3. Case 3. Computed tomography scan obtained 18 months postoperatively showing solid bony fusion across the affected disc space.

such as Staphylococcus aureus, and the pathophysiology may be different.

Our case series was a continuum of the known posterior approach but focused on stabilization rather than debridement of the pus as being the most important part of therapy. Indeed, we stabilized above and below the infected area and left the infected vertebral level alone, aside from decompression of the neural elements. There are data suggesting that rigid immobilization of an infected area is critical in bone healing. The greatest quantity of these data come from open tibia fractures and infected tibial fracture nonunions. Multiple studies have shown that, in these very challenging scenarios, rigid fixation of the fracture results in improved outcomes, even when metallic or cement implants are placed into an infected area. ${ }^{910}$ Similarly, Govender et al. found a lower rate of infection in high-grade open tibia fractures when using BMP-2 in a high-level clinical trial. Their theory for this finding was that a shorter time to union and improved blood flow from early healing results in prevention of infection. ${ }^{2}$ Our results suggest that this approach can be successful in the spine with all infections resolved or suppressed and a very high likelihood of improvement in neurological function (see Figs. 1-3 for an illustrative case).

Our postoperative complications were similar in type to those reported in other posterior approach studies and included hardware failure, hematoma, and superficial wound infection. Our complication rates were impossible to compare with other studies because of the small number of pa- tients. Our population was, as expected, a relatively sick and complex patient population, with an average Charlson Comorbidity Index of 2 and an average American Society of Anesthesiologists score of 2.8. Paradoxically, the average body mass index was elevated at 28 , but almost all of our patients' preoperative serum albumin levels were below $3 \mathrm{~g} / \mathrm{dl}$. These patients were very sick, nutritionally depleted, and many of them were neurologically in extremis. It was our belief that their physiological reserve could not sustain a combined anterior/posterior approach for formal debridement. Despite this severity of disease, the mortality rate in this population was relatively low. No patients died within 12 months of surgery. Four patients died during the follow-up period, but of severe medical comorbidities rather than spinal or infectious complications.

Our study is generally homogeneous in treatment method. Almost all of our patients were treated with posterior-only surgery, but in 1 case, we did perform an anterior/posterior approach. The patient in Case 7 was a 52-year-old woman with a history of diabetes mellitus who underwent multiple laminectomies for an epidural abscess at L4-5. After several repeat laminectomies with recurrence of epidural infection and progressive neurological deficit, she was scheduled for posterior long-segment fixation and revision laminectomy. The night before surgery she went into cardiopulmonary arrest secondary to massive myocardial infarction and mitral valve rupture and required intraaortic balloon pump placement. She then underwent coronary artery bypass grafting 3 times and valve replacement. She was treated with intravenous antibiotics for her spinal infection without further surgery, until about 4 weeks later when she developed cauda equina syndrome and recurrent infection. At that time she was treated with posterior decompression and stabilization. Given the recalcitrant nature of her disease and the fact that her medical comorbidities had delayed our initial treatment, approximately 6 weeks later she underwent anterior discectomy and fusion for debridement of her disc space. She continues to be followed by us, with resolution of her cauda equina syndrome but persistent leg weakness and no infectious sequelae, now 5 years after surgery. We feel her case is somewhat exceptional in our case series.

It is difficult to objectively quantify the extent to which the multidisciplinary nature of our infectious diseases/ spine team contributed to the successful outcomes of our patients. We strongly believe, however, that reproduction of our results depends on such a collaborative approach.

There are a number of limitations of our study, of course. First of all, this is a retrospective case series, without a control population. While it is possible that we were selective in our use of this approach, this series includes all cases meeting the inclusion criteria and treated by 2 of us over a 4-year period. Neither of us referred any patients to other colleagues for different surgical management. Our patient numbers are not large. Without more patients, comparing our complication rates to those historically reported is not conclusive. We deliberately sought to define a population that included all patients treated in the same manner. We included only patients of the 2 authors because their practice contained patients exclusively treated in the described manner, with the exception of Case 7 described 
above. While this restricted our numbers, it allowed us to maintain a relatively homogeneous patient population in terms of surgical management. There is some heterogeneity in terms of indications for surgery. Levels of infection ranged from the upper cervical spine through the lumbosacral junction. About half of our patients underwent urgent stabilization at the time of diagnosis of spinal infection for either actual or expectant neurological injury. About half of our population underwent fixation after prior management led to a combination of progressive bony instability with continued infection, evolving neurological deficit, or persistent systemic signs of infection with progression of spinal disease on advanced imaging. This heterogeneity represents clinical practice in that many cases of spinal infection can be treated without surgery, and only patients with progressive deficit, progressive instability, or failure of nonoperative treatment require surgery. When in doubt, we attempted to treat either without surgery or with laminectomy alone, and we proceeded to fixation when this failed.

Our follow-up rate for this condition was relatively good, but less than would be ideal. We lost 1 patient almost immediately after surgery. Two additional patients were seen at more than 1 year after surgery, but not longer. Four patients did not have complete antibiotic therapy data: 3 because they underwent outside infectious disease management and their records were not available to us for review, and the one patient who was lost to follow-up before a final plan was established. All other patients were contacted either in clinic or by phone for long-term followup. It is possible that one of our lost patients had a treatment failure, but as the only academic medical center and 1 of 2 tertiary care spine centers within our state, we believe it unlikely that these patients presented with a recurrent infection without the treating institution contacting us.

Lastly, it is possible that some of our cases might have been treated without surgery or with surgery without fixation. However, our selection criteria for surgery were fairly strict and required either a progressive or profound neurological deficit or a failure of prolonged antibiotic therapy with evidence of bony instability on radiography or CT scanning. It was our opinion at the time, and remains our opinion today, that all of these patients had clear indications for surgical treatment with stabilization and could not have been treated with either antibiotics and external bracing or simple decompression and antibiotics alone. In fact, a number of our patients (5 of 15) had initial treatment with laminectomy and developed recurrent symptoms as the indication for stabilization.

\section{Conclusions}

Long-segment fixation without formal debridement, when combined with aggressive antibiotic therapy and a multidisciplinary team approach, was an effective method of managing vertebral column infection in a challenging and complex patient population.

\section{Acknowledgments}

We would like to thank Ms. Sabina Blizzard, B.A., and Ms. Natalie Zusman, B.S., for their work on this study.

\section{Disclosure}

Each author certifies that he or she has not received any payments of benefits from a commercial entity related to this work. Dr. Hart is a consultant for DePuy, Medtronic, and Globus. He owns stock in Spine Connect. He is a patent holder with OHSU, and reports receiving non-study-related clinical or research support from ISSGF and Medtronic. He receives royalties from Seaspine and DePuy. Dr. Ching is a consultant for DePuy Synthes Spine, NuVasive Spine, and Atlas Spine. He reports receiving a departmental education grant from Globus Spine.

Author contributions to the study and manuscript preparation include the following. Conception and design: Ching, Yoo, Hart. Drafting the article: Ching, Mohamed, Ragel, Hiratzka, Hamilton, Barnes. Critically revising the article: Ching, Mohamed, Yoo, Hart. Reviewed submitted version of manuscript: Ching, Mohamed. Approved the final version of the manuscript on behalf of all authors: Ching. Administrative/technical/material support: Ching, Mohamed. Study supervision: Ching, Yoo.

\section{References}

1. Eismont FJ, Bohlman HH, Soni PL, Goldberg VM, Freehafer AA: Pyogenic and fungal vertebral osteomyelitis with paralysis. J Bone Joint Surg Am 65:19-29, 1983

2. Govender S, Csimma C, Genant HK, Valentin-Opran A, Amit Y, Arbel R, et al: Recombinant human bone morphogenetic protein-2 for treatment of open tibial fractures: a prospective, controlled, randomized study of four hundred and fifty patients. J Bone Joint Surg Am 84-A:2123-2134, 2002

3. Hadjipavlou AG, Mader JT, Necessary JT, Muffoletto AJ: Hematogenous pyogenic spinal infections and their surgical management. Spine (Phila Pa 1976) 25:1668-1679, 2000

4. Hee HT, Majd ME, Holt RT, Pienkowski D: Better treatment of vertebral osteomyelitis using posterior stabilization and titanium mesh cages. J Spinal Disord Tech 15:149-156, 2002

5. Kaufman DM, Kaplan JG, Litman N: Infectious agents in spinal epidural abscesses. Neurology 30:844-850, 1980

6. Lee JS, Moon KP, Kim SJ, Suh KT: Posterior lumbar interbody fusion and posterior instrumentation in the surgical management of lumbar tuberculous spondylitis. J Bone Joint Surg Br 89:210-214, 2007

7. Lee MC, Wang MY, Fessler RG, Liauw J, Kim DH: Instrumentation in patients with spinal infection. Neurosurg Focus 17(6):E7, 2004

8. Lu DC, Wang V, Chou D: The use of allograft or autograft and expandable titanium cages for the treatment of vertebral osteomyelitis. Neurosurgery 64:122-130, 2009

9. Patzakis MJ, Wilkins J, Wiss DA: Infection following intramedullary nailing of long bones. Diagnosis and management. Clin Orthop Relat Res (212):182-191, 1986

10. Sancineto CF, Barla JD: Treatment of long bone osteomyelitis with a mechanically stable intramedullar antibiotic dispenser: nineteen consecutive cases with a minimum of 12 months follow-up. J Trauma 65:1416-1420, 2008

11. Sofianos D, Patel AA: Vertebral osteomyelitis. Contemp Spine Surg 11:1-8, 2010

12. Zimmerli W: Clinical practice. Vertebral osteomyelitis. $\mathbf{N}$ Engl J Med 362:1022-1029, 2010

Manuscript submitted April 15, 2014.

Accepted June 2, 2014.

Please include this information when citing this paper: DOI: 10.3171/2014.6.FOCUS14142.

Address correspondence to: Alexander C. Ching, M.D., Department of Orthopedics and Rehabilitation, Oregon Health \& Science University, 3181 SW Sam Jackson Parkway Rd., OP31, Portland, OR 97239. email: chinga@ohsu.edu. 